\title{
Perfil epidemiológico das principais doenças veiculadas por alimentos
}

\author{
Epidemiological profile of the main diseases veiculated by foods \\ Perfil epidemiológico de las principales enfermedades realizadas por los alimentos
}

Recebido: 05/08/2021 | Revisado: 10/08/2021 | Aceito: 13/08/2021 | Publicado: 15/08/2021

\author{
Bruna Silva Araújo \\ ORCID: https://orcid.org/0000-0003-4140-8224 \\ Universidade Salvador, Brasil \\ E-mail: bruna_bsaraujo@hotmail.com \\ Sandra Rojas Duailibi \\ ORCID: https://orcid.org/0000-0001-7373-0950 \\ Universidade Estácio de Sá, Brasil \\ E-mail:srdconsultoria@gmail.com \\ Lorena Rachid \\ ORCID: https://orcid.org/0000-0002-6728-5261 \\ Universidade Federal da Bahia, Brasil \\ E-mail: lore_rachid@hotmail.com
}

\begin{abstract}
Resumo
A incidência de surtos veiculados por alimentos tem ganhado uma proporção elevada e estima-se que tenha mais casos não notificados. O objetivo do estudo é elucidar o perfil epidemiológico dos surtos de DVA no Brasil com base nos dados do Manual Integrado de Vigilância, Prevenção e Controle de Doenças Transmitidas por Alimentos. Assim, o estudo está estruturado pela apresentação dos elementos de investigação das DVA, as principais categorias e os locais de produção dos elementos envolvidos nos surtos e por fim, a relação dos principais micro-organismos encontrados nas análises dos surtos notificados e seus efeitos à saúde humana. Trata-se de um estudo descritivo, epidemiológico, documental com abordagem quantitativa e com as variáveis analisadas: alimentos e surtos 2016 e 2017, sinais e sintomas 2016 e 2017, perfil epidemiológico dos doentes 2016 e 2017, surtos e regiões do Brasil 2016 e 2017 e Micro-organismos veiculado aos alimentos 2016 e 2017. Ao analisar o local de produção dos alimentos, verificou-se que os surtos ocorrem em sua maioria nas residências e estabelecimentos comerciais. Desta forma, fica evidente a necessidade de mais estudos e de se buscar alternativas públicas para mudança comportamental da população e a inclusão de nutricionistas nos estabelecimentos comerciais e industriais.
\end{abstract}

Palavras-chave: Perfil epidemiológico; Surto; Alimento; Contaminação; DVA; Micro-organismos causadores de DVA; Boas práticas no preparo de alimentos.

\begin{abstract}
The incidence of foodborne outbreaks has gained a high proportion and it is estimated that there are more unreported cases. The aim of the study is to elucidate the epidemiological profile of VAD outbreaks in Brazil based on data from the Integrated Manual for Surveillance, Prevention and Control of Foodborne Diseases. Thus, the study is structured by the presentation of the elements of investigation of VAD, the main categories and production sites of the elements involved in the outbreaks and, finally, the list of the main microorganisms found in the analyzes of the reported outbreaks and their health effects human. This is a descriptive, epidemiological, documentary study with a quantitative approach and with the variables analyzed: food and outbreaks 2016 and 2017, signs and symptoms 2016 and 2017 , epidemiological profile of patients in 2016 and 2017, outbreaks and regions of Brazil 2016 and 2017 and Microorganisms transmitted to food 2016 and 2017. When analyzing the place of food production, it was found that the outbreaks occur mostly in homes and commercial establishments. Thus, the need for further studies and public alternatives to change the population's behavior and the inclusion of nutritionists in commercial and industrial establishments is evident.

Keywords: Epidemiological profile; Outbreak; Food; Contamination; DVA;Microorganisms causing DVA; Good practices in food preparation.

\section{Resumem}

La incidencia de brotes de enfermedades transmitidas por alimentos ha ganado una proporción elevada y se estima que hay más casos no denunciados. El objetivo del estudio es dilucidar el perfil epidemiológico de los brotes de DVA en Brasil a partir de los datos del Manual Integrado de Vigilancia, Prevención y Control de Enfermedades Transmitidas por Alimentos. Así, el estudio se estructura mediante la presentación de los elementos de investigación de la DAV, las principales categorías y sitios de producción de los elementos involucrados en los brotes y, finalmente, la lista de los principales microorganismos encontrados en los análisis de los brotes reportados y sus efectos sobre la salud humana.
\end{abstract}


Se trata de un estudio descriptivo, epidemiológico, documental con enfoque cuantitativo y con las variables analizadas: alimentos y brotes 2016 y 2017, signos y síntomas 2016 y 2017, perfil epidemiológico de los pacientes en 2016 y 2017 , brotes y regiones de Brasil 2016 y 2017. y Microorganismos transmitidos a los alimentos 2016 y 2017. Al analizar el lugar de producción de alimentos, se encontró que los brotes ocurren mayoritariamente en hogares y establecimientos comerciales. Así, se evidencia la necesidad de mayores estudios y alternativas públicas para cambiar el comportamiento de la población y la inclusión de nutricionistas en los establecimientos comerciales e industriales.

Palabras clave: Perfil epidemiológico; Brote; Comida; Contaminación; DVA; Microorganismos causantes de DAV; Buenas prácticas en la preparación de alimentos.

\section{Introdução}

Atualmente, muitas enfermidades mundiais são de origem alimentar, os alimentos e a água são indispensáveis à vida humana, mas também podem sera causa de diversas morbidades, já que estão suscetíveis a contaminações (Câmara, Gomes, Matuk, \& Szarfarc, 2014).

As síndromes, resultantes da ingestão de alimentos contaminados por esses micro-organismos são conhecidas como Doenças Transmitidas por Alimentos (DTA) ou Doenças Veiculadas por Alimentos (DVA) (Buzby, Roberts, \& Tanya. 2009).

As doenças veiculadas por alimentos (DVA) tem sua origem física, química e microbiológica, que permeiam todas as etapas da cadeia alimentar e constituem um dos problemas de saúde pública mais frequente do mundo. São causadas por agentes etiológicos, principalmente microrganismos, os quais penetram no organismo humano através da ingestão de água e alimentos contaminados (Welker et al., 2010).

Geralmente sintomas digestivos estão associados à síndrome e se constituem de anorexia, náuseas, vômitos e/ou diarreia, acompanhada ou não de febre, porém, não são os únicos podem vir associados à sintomas extra intestinais em diferentes órgãos como: rins, fígado e sistema nervoso central relacionados à ingestão de alimentos ou água contaminados (Ministério da Saúde, 2010).

Entre as causas mais frequentes de contaminação dos alimentos, destacam-se a manipulação e a conservação inadequadas dos mesmos, além da contaminação cruzada entre produtos crus e processados (Mürmann, Santos, Longaray, Both, \& Cardoso, M. 2008).

O presente estudo tem por objetivo apresentar um levantamento de dados epidemiológicos de surtos alimentares, caracterizando os locais de ocorrência e seus agentes etiológicos nos anos de 2007 a 2017 no Brasil.

Doenças Veiculadas por alimentos (DVA) representam um entrave significativo ao desenvolvimento socioeconômico em todo o mundo, e nem sempre há dados que permitam conhecer e identificar as causas dessas doenças, contudo a Organização Mundial da Saúde estima que, sejam acometidos os surtos de origem microbiológica, atribuindo esse fato a manipulação e condições higiênicas sanitárias inadequadas (Santos, 2014).

Apesar das lacunas de dados e limitações, evidencia-se que a ocorrência global de DTA é alta e afeta indivíduos de todas as idades, mas especialmente crianças menores de cinco anos de idade, idosos, com problemas imunológicos e que vivem em sub-regiões de baixa renda do mundo, como as crianças e idosos, que mais sofrem com o problema. Segundo estimativas da Agência das Nações Unidas, o número de mortes ao ano por DVA, em todo o mundo, é de 420 mil. As afetadas ficam doentes após consumirem alimentos contaminados por bactérias, vírus, parasitas, toxinas ou produtos químicos, conforme os dados o sistema de saúde deverá ter mais atenção e cuidado (Who, 2019).

\section{Investigação da DVA no Brasil}

A DVA pode dar origem a surtos definidos por episódios nos quais duas ou mais pessoas apresentam, em um mesmo período de tempo, sinais esintomas análogos após a ingestão de alimento de mesma procedência considerado contaminado por evidência clínica, epidemiológica e/oulaboratorial (Marchi, Baggio, Teo, \& Busato, 2011). 
A DVA pode ser causada por: bactérias, vírus, parasitas, toxinas, príons, agrotóxicos, produtos químicos e metais pesados. O quadro clínico depende doagente etiológico envolvido e varia desde leve desconforto intestinal até quadros extremamente sérios, podendo levar a desidratação grave, diarreia sanguinolenta e insuficiência renal aguda (Ministério da Saúde, 2016).

Nos últimos anos, a investigação de surtos de DVA no Brasil vem se desenvolvendo rapidamente. O problema da subnotificação de surtos existe e continua presente, atualmente, o Brasil possui uma série de ferramentas e informações sobre surtos notificados, e de procedimentos para a investigação dos mesmos a qual tem sido utilizada para estabelecer estratégias de prevenção de novas DVA (Sirtoli \& Comarella, 2018).

A investigação epidemiológica das DVA tem o objetivo de coletar earmazenar informações necessárias ao controle do surto, identificar as fontes de transmissão e os fatores de riscos associados ao surto, bem como diagnosticar a doença, identificar seus agentes etiológicos e propor medidas decontrole e prevenção, contudo existe uma série de medidas descritas na figura acima para a detecção e finalização do surto (Fischer, 2013).

\section{Metodologia}

Trata-se de estudo descritivo, epidemiológico, documental com abordagem quantitativa, baseado em dados secundários referentes aos casos de DVA notificados no Brasil, entre o período 2007 a 2017. De acordo com Rouquayrol \& Silva, (2018) os estudos epidemiológicos permitem demonstrar a distribuição dos determinantes das doenças ou condições relacionadas à saúde em populações especificadas, enquanto o cunho descritivo permite a descrição da análise de dados em um período temporal determinado. Já a abordagem quantitativa é utilizada em levantamento de dados matemáticos por meio da utilização de porcentagens (Pereira et al., 2018).

A coleta dos dados ocorreu no ano de 2018, por meio eletrônico, a partir do Ministério da saúde, base de dados disponibilizada pelo Manual Integrado de Vigilância, Prevenção, Controle de Doenças transmitidas por Alimentos.

As variáveis analisadas foram: Alimentos e surtos 2016 e 2017, sinais e sintomas 2016 e 2017, perfil epidemiológico dos doentes 2016 e 2017, alimentos e surtos 2016 e 2017, surtos e regiões do Brasil 2016 e 2017 e Micro-organismos veiculado aos alimentos 2016 e 2017.

\section{Resultados e Discussão}

De acordo com a Portaria n 2.472, de 31 de agosto de 2010 (Ministério da Saúde, 2010), todo surto de DVA deverá ser notificado às autoridades locais de saúde (Secretária Municipal de Saúde) e investigado imediatamente. A unidade de saúde notificadora deve utilizar a ficha de notificação/investigação do Sistema de Informação de Agravos de Notificação (SINAN), encaminhando-a para ser processada conforme o fluxo estabelecido pela Secretaria Municipal de Saúde descrito na Figura 1 (Ministério da Saúde, 2010). 
Figura 1: Investigação de Surtos e DVA.

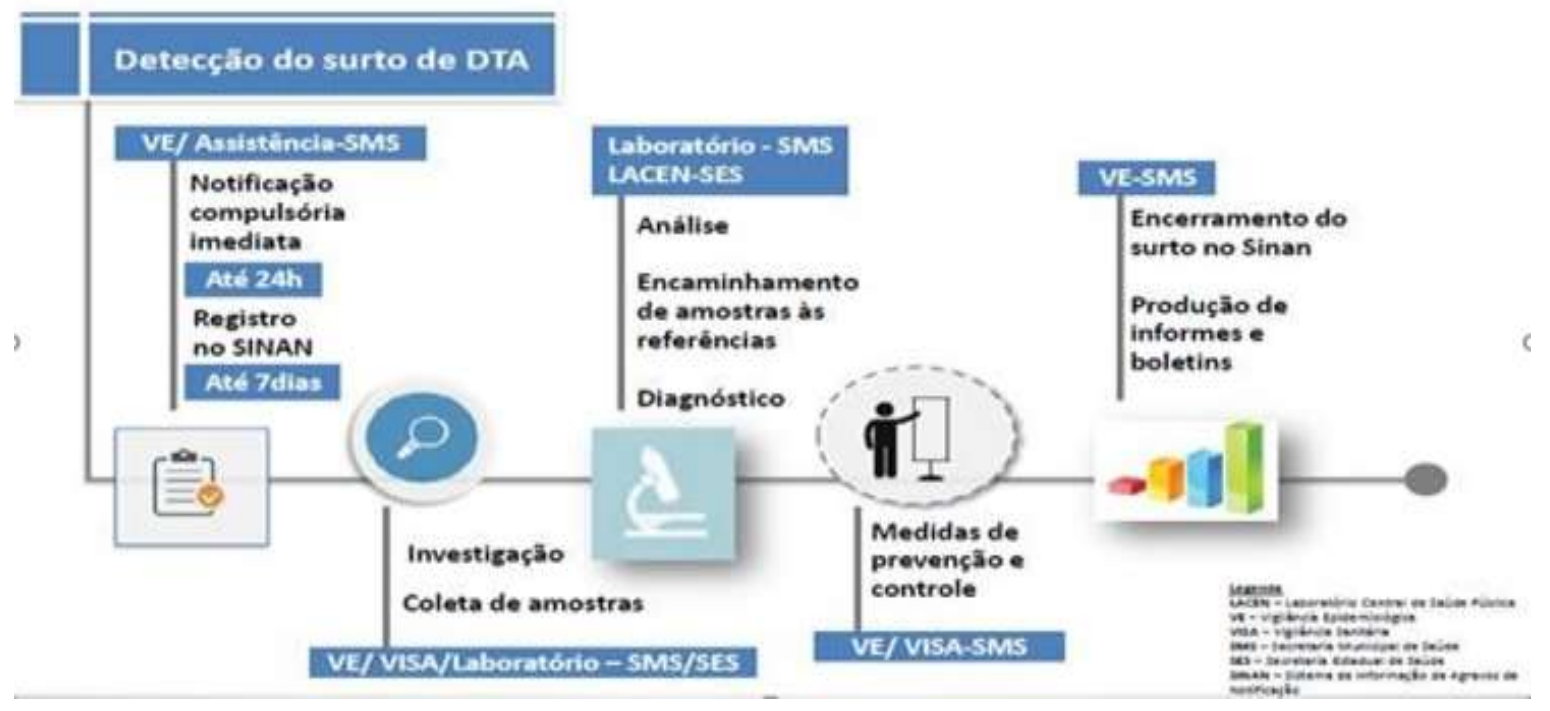

Fonte: Ministério da Saúde. Manual Integrado de Vigilância, Prevenção e Controle de Doenças Transmitidas por Alimentos, (2016)

Devido ao aumento dos casos de DVA no país, a SVS/MS desenvolveu o Sistema Nacional de Vigilância Epidemiológica das Doenças Transmitidaspor Alimentos (VE-DTA). Implantado em 1999, em parceria com a ANVISA, Ministério da Agricultura, Pecuária e Abastecimento (MAPA) e Instituto Pan- Americano de Alimentos da Organização PanAmericana de Saúde (OPAS). O Sistema VE-DTA tem como objetivos reduzir a incidência das DVAS no Brasil, identificar a suspeita da ocorrência de um evento na população e indicar os riscos aos quais as pessoas estão sujeitas, contribuindo, assim, para a identificação da realidade epidemiológica de determinada área geográfica (Ministério da Saúde, 2008).

\subsection{Surtos de DVA confirmados no Brasil}

A Segurança dos alimentos trata da confirmação de que os alimentos não serão vínculos de doenças aos seus consumidores, ressaltando a importância das condições higiênicas do local de produção e manipulação do alimento, pois interferem diretamente na qualidade microbiológica do mesmo por serem considerados como pontos de contaminação (Alves, Giarreta, \& Costa, 2012).

Na maioria das vezes os próprios manipuladores são frequentemente disseminadores de agentes patológicos e favorecem a produção de algumas toxinas em alimentos como higiene pessoal precária, preparos de alimentos feitos com muita antecedência, alimentos com aquecimento e cocçãoinadequados de alguns alimentos; mal uso de pratos para servir os alimentos e refrigeração inadequada (Junior et al., 2008).

Nessa linha, estudos recentes evidenciam que não existe associação entre o nível de conhecimento, as atitudes e as práticas dos manipuladores de alimentos, pois o conhecimento e os treinamentos em segurança alimentar nãoasseguram que as práticas sejam devidamente realizadas, sendo importante a formação contínua dos manipuladores de alimentos para a produção de alimentos seguros (Soares, Almeida, Cerqueira, Carvalho, \& Nunes, 2012).

Os casos de Doenças Veiculadas por Alimentos (DVA) detectados nos estabelecimentos comerciais e nas residências são resultantes de práticasincorretas de manipulação de alimentos (Mello, Gama, Marin, \& Colares, 2010). Nesse contexto, alguns autores relatam a existência de fatores externos e internos que limitama adoção das Boas Práticas de Manipulação, tais como: falta de tempo, inexistência de recursos materiais, falta de responsabilidade, falta de apoio daschefias e dos próprios 
colegas de trabalho, falta de comprometimento dasorganizações, falta de formação e carência de pessoal (Tokuç, Ekuklu, Berberogu, Bilge, \& Dedeler, 2009; Melo et al., 2018)

Gráfico 1: Perfil Epidemiológico dos Surtos e DVA em 2016.

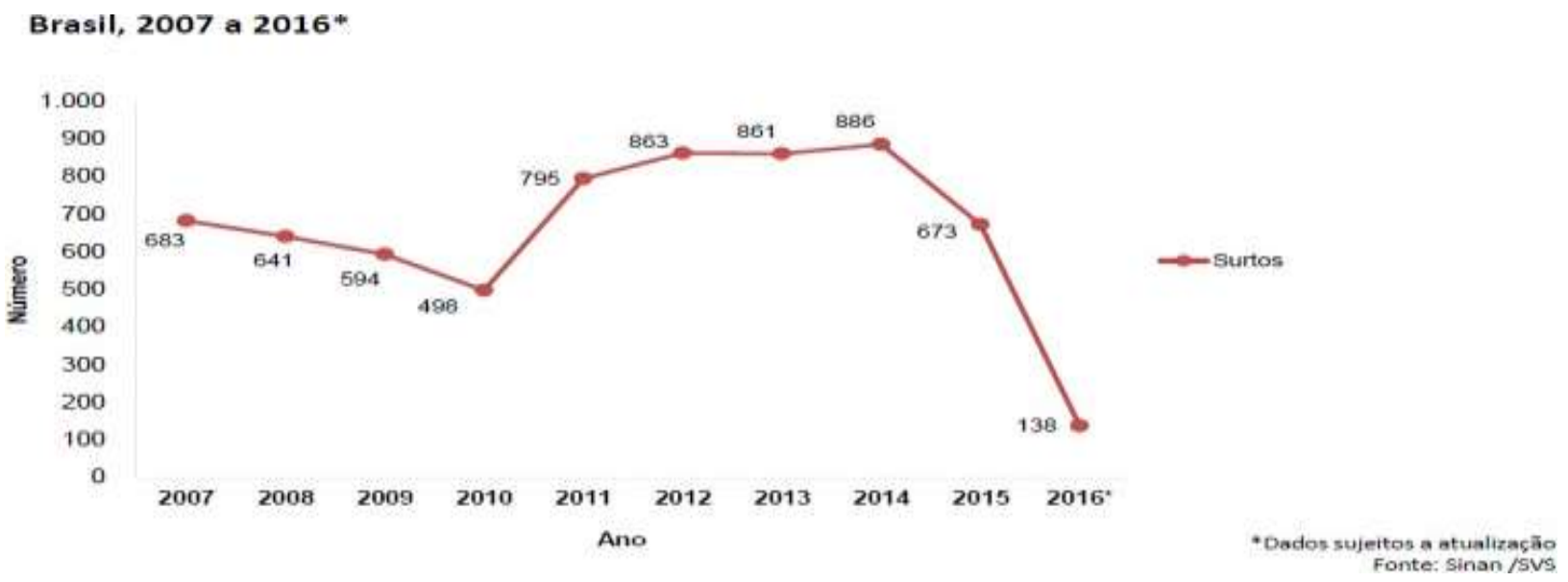

rasil, 2007 a $2016^{*}$

Fonte: Ministério da Saúde. Manual Integrado de Vigilância, Prevenção eControle de Doenças Transmitidas por Alimentos, (2016).

Gráfico 2: Perfil Epidemiológico dos Surtos e DVA em 2017.

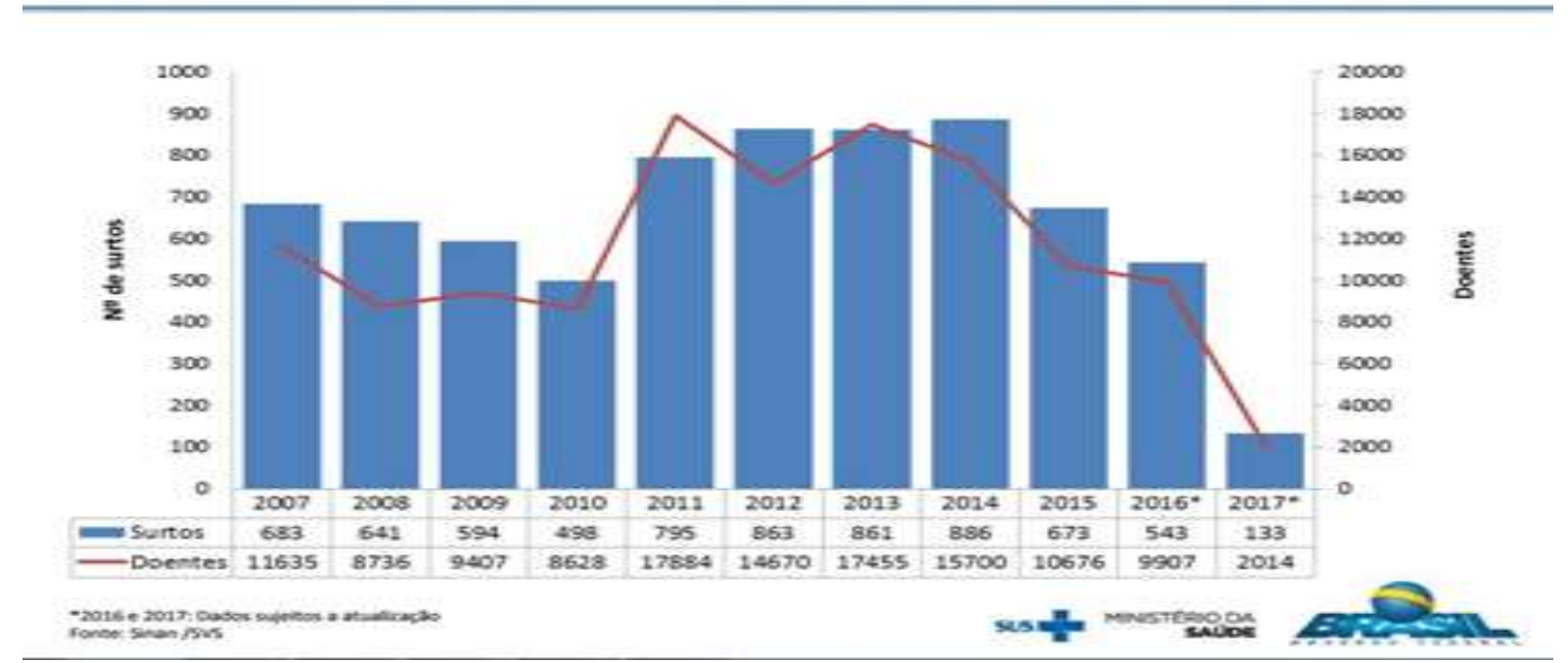

Fonte: Ministério da Saúde. Manual Integrado de Vigilância, Prevenção e Controle de Doenças Transmitidas por Alimentos (2017).

Nos gráficos de 2016 a 2017, 138 surtos foram confirmados por investigação criteriosa no ano de 2016, porém após a publicação houve um acréscimo de 405 casos, como identificamos no gráfico 2, contudo em 2017 até a data de publicação, houve 133 casos, no Gráfico 1 não relata números de doentes, logo não tem possibilidade de comparações. 
Gráfico 3: Perfil Epidemiológico dos doentes em 2016.

\section{Brasil, 2007 a 2016*}
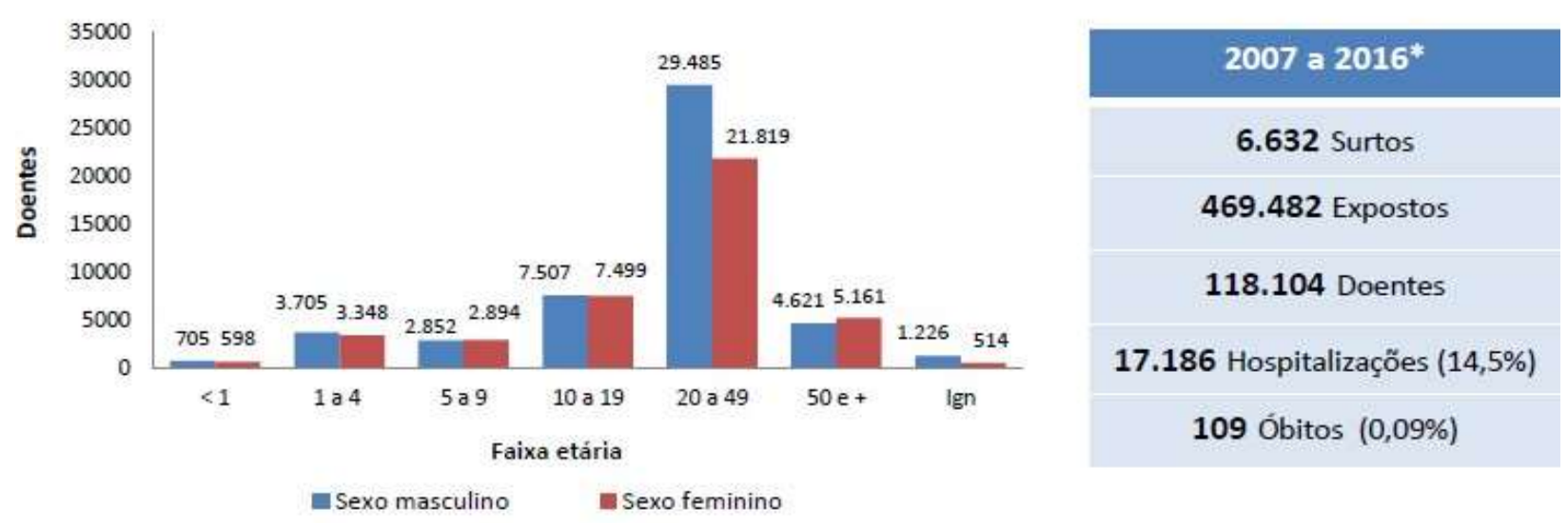

- Dados sujeitos a atualização Fonte: Sinan/SVS

Fonte: Ministério da Saúde. Manual Integrado de Vigilância, Prevenção e Controle de Doenças Transmitidas por Alimentos, (2016).

Gráfico 4: Perfil Epidemiológico dos doentes em 2017.

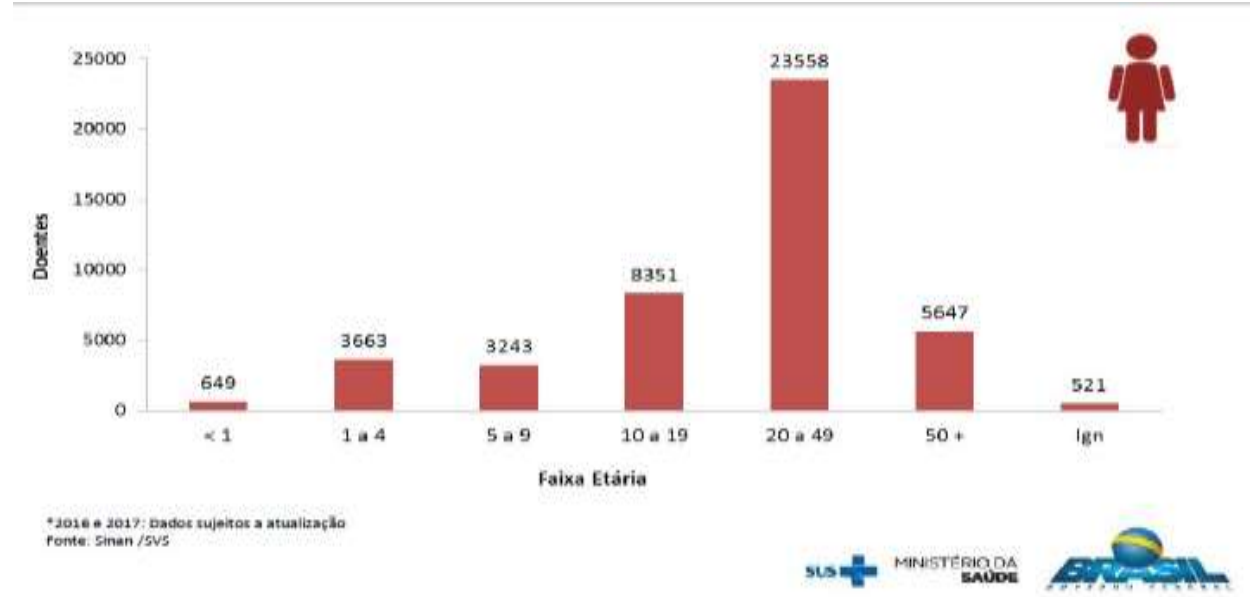

Fonte: Ministério da Saúde. Manual Integrado de Vigilância, Prevenção e Controle de Doenças Transmitidas por Alimentos, (2017).

Gráfico 5: Perfil Epidemiológico dos doentes em 2017.

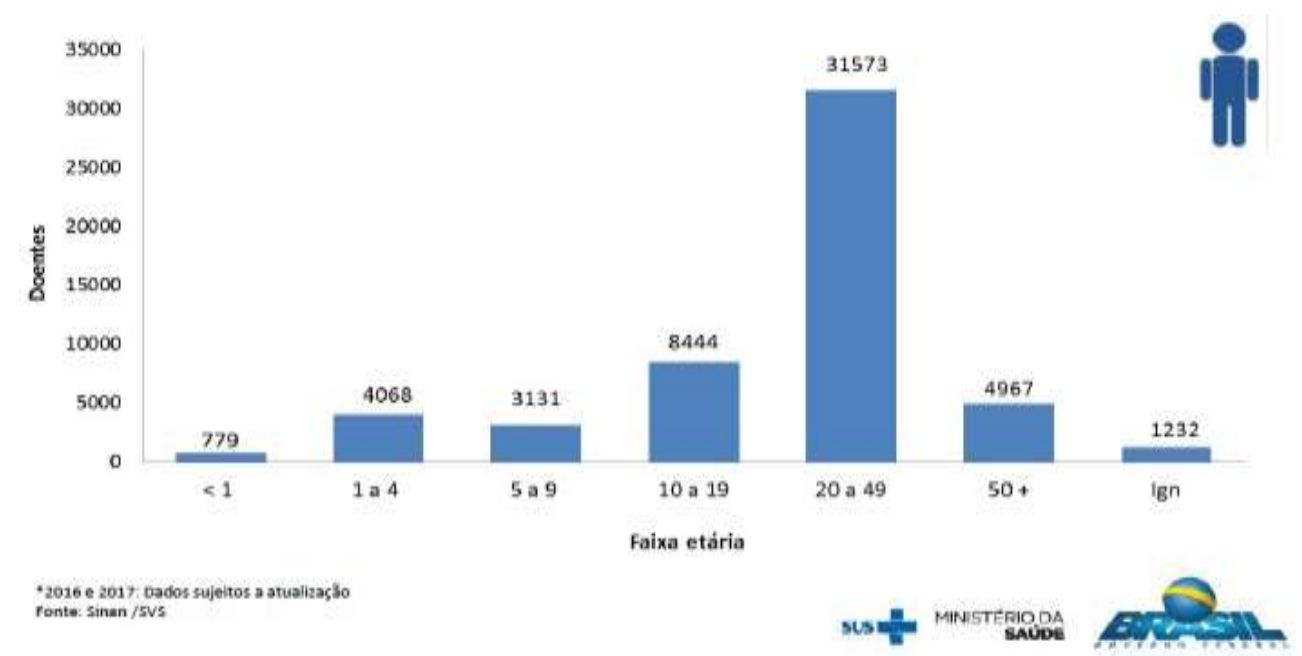

Fonte: Ministério da Saúde. Manual Integrado de Vigilância, Prevenção e Controle de Doenças Transmitidas por Alimentos, (2017). 
Na comparação dos gráficos 3, 4 e 5 foi identificada a relação da faixa etária e os sexos com maior índice de doentes, onde as faixas em foco foram 10 a 19 anos e 20 a 49 anos de ambos os sexos, o sexo masculino se mostrou com maior índice de doentes onde em 2016 na faixa de 10 a 19 em comparação com as do sexo feminino houve um aumento de 8 doentes, já em 2017 nessa mesma faixa já identificados um aumento de 93 doentes, já na faixa etária de 20 a 49 anos foram identificados 7.666 doentes a mais em relação ao sexo feminino, em 2017 a situação se repete houve um aumento de 8.015 comparados entre o ano de 2016 e 2017.

Gráfico 6: Sinais e Sintomas em 2016.

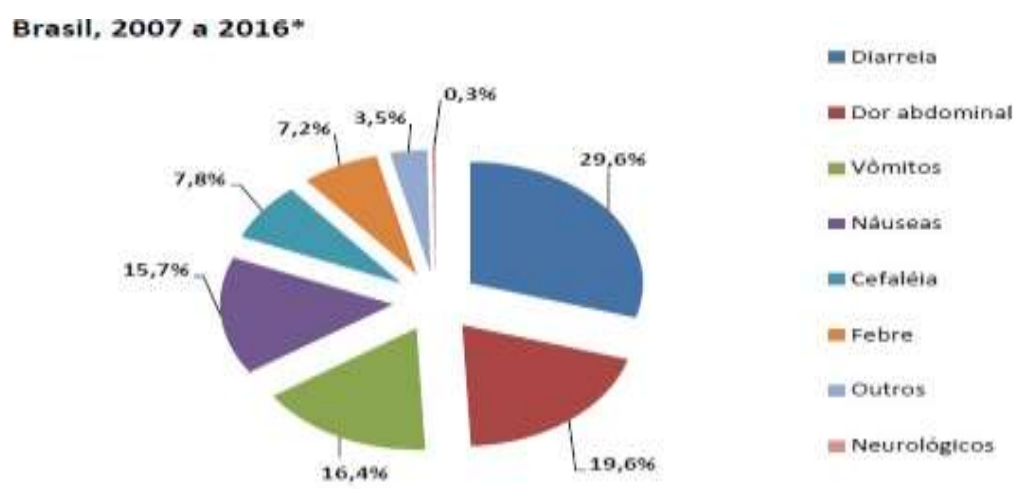

Fonte: Ministério da Saúde. Manual Integrado de Vigilância, Prevenção e Controle de Doenças Transmitidas por Alimentos, (2016).

Gráfico 7: Sinais e Sintomas em 2017.

\section{Proporção de sinais e sintomas em surtos de DTA. Brasil, 2007 a 2017*}
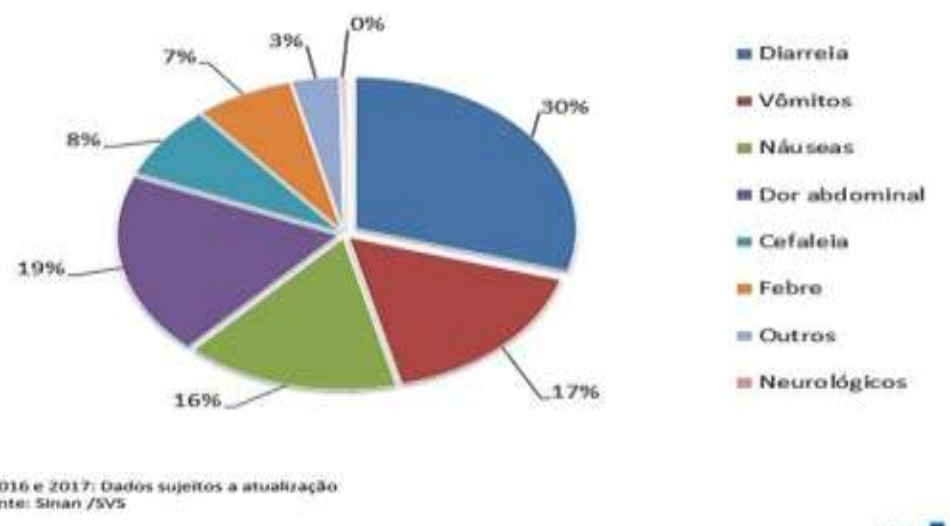

2036 e 2017 basis
ronte: sinan /svis
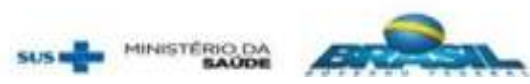

Fonte: Ministério da Saúde. Manual Integrado de Vigilância, Prevenção e Controle de Doenças Transmitidas por Alimentos, (2017).

Os sintomas mais identificados de DVA incluem dor de estômago, náusea, vômitos, diarreia e, por vezes, febre. Na maioria dos quadros clínicos, existe um período de incubação para os primeiros sintomas aparecerem, podendo se manifestar em poucas horas ou em até mais de cinco dias, dependendo do estado físico do paciente, do tipo de micro-organismo ou toxina ingerida ou suas quantidades no alimento. Conforme o agente etiológico envolvido, o quadro clínico pode ser mais grave e prolongado, apresentando desidratação grave, diarreia sanguinolenta, insuficiência renal aguda, insuficiência respiratória, demências, meningite e aborto espontâneo (Forsythe, 2010).

Na comparação dos Gráficos 6 e 7, de 2016 a 2017 houve aumentos nos itens diarreia: 0,4\%; Vômito: 0,6\%; Náuseas: 0,3\%; Cefaleia: 0,2\%, ocorrendo a diminuição nos quesitos Dor abdominal: 0,6\%; Febre: 0,2\%; Neurológicos: 0,3\% e Outros: $0,2 \%$, são resultados pouco significativos emnível de sinais e sintomas. 


\subsection{Alimentos e locais de produção envolvidos nos surtos de DVA no Brasil}

Gráfico 8: Alimentos e surtos em 2016.

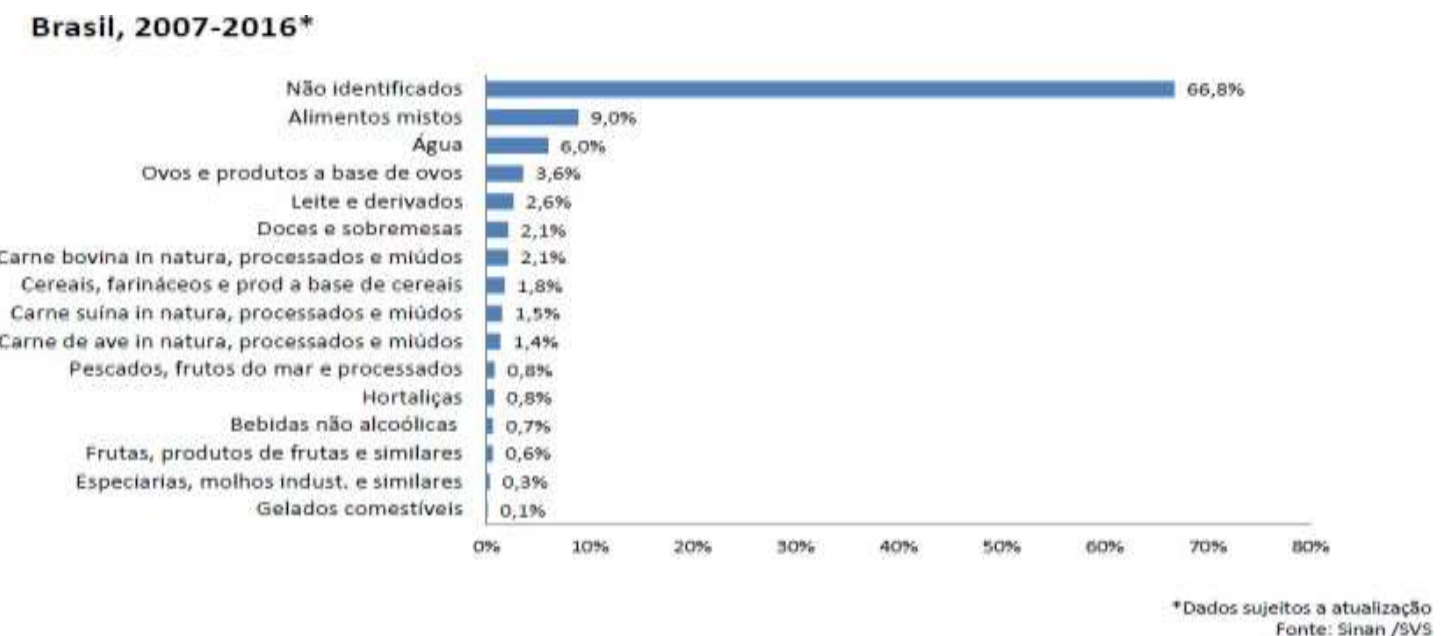

Fonte: Ministério da Saúde. Manual Integrado de Vigilância, Prevenção e Controle de Doenças Transmitidas por Alimentos, (2016).

Gráfico 9: Alimentos e surtos em 2017.

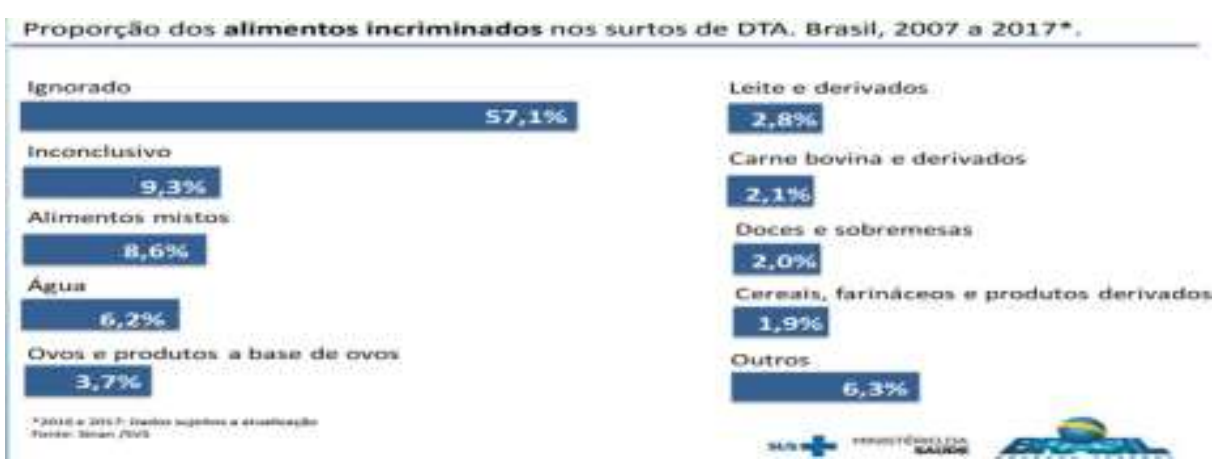

Fonte: Ministério da Saúde. Manual Integrado de Vigilância, Prevenção e Controle de Doenças Transmitidas por Alimentos, (2017).

Na comparação entre os gráficos 8 e 9 referentes aos surtos não identificados, Nascimento (2013), relata que o elevado índice pode estar relacionado à demora na notificação do surto, dificuldade em entrar em contato com os comensais envolvidos, bem como a falta de informações precisas sobreas refeições realizadas e a procedência delas.

O ovo pode estar contaminado na superfície externa, devido às fezes da galinha, o que pode ocorrer pela entrada através da casca por uma higienização inadequada ou da contaminação direta do conteúdo dos ovos, antes da oviposição e/ ou internamente, proveniente de infecção dos órgãos reprodutivos (Gantois et al., 2009; Zhang, Brown, \& Hammack, 2013).

Cabe destacar que a própria maionese pode propiciar elevada multiplicação microbiana, tanto a caseira como a industrializada, uma vez que tanto o ovo como a batata apresentam características de meio de cultura, ou seja, acabam sendo alimento para as bactérias se multiplicarem, devido às suas propriedades nutritivas (intrínsecas) (Wagner, Silveira, \& Tondo, 2014; Capalonga et al., 2014).

A água para o consumo humano e para manipulação de alimentos sem tratamento adequado representa um dos principais veículos de parasitas, microrganismos causadores de doenças, tornando-se um importante elemento de risco à saúde da população que a consome. Dentre os patógenos mais comuns incluem-se Salmonella spp., Shigella spp., Escherichia coli e Campylobacter (Guilherme, \& Esteves,.2017; Klein, Bisognin, \& Figueiredo, S.2017). 


\subsection{Surtos e região do Brasil}

Gráfico 10: Frequência de Surtos de DVA por região em 2016.

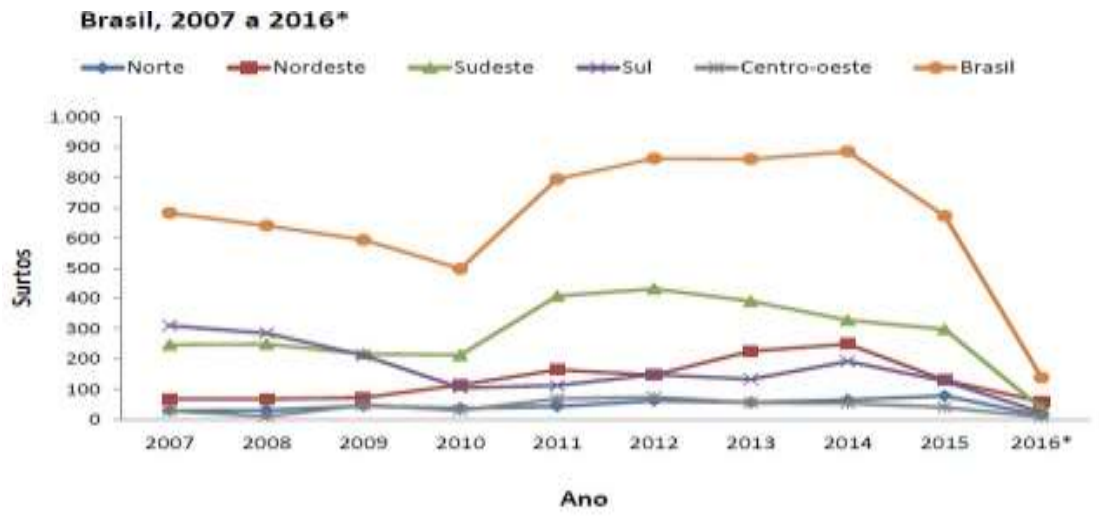

Fonte: Ministério da Saúde. Manual Integrado de Vigilância, Prevenção e Controle de Doenças Transmitidas por Alimentos, (2016).

Gráfico 11: Frequência de Surtos de DVA por região em 2017.

Frequência dos surtos de DTA por região. Brasil, 2007 a 2017*.

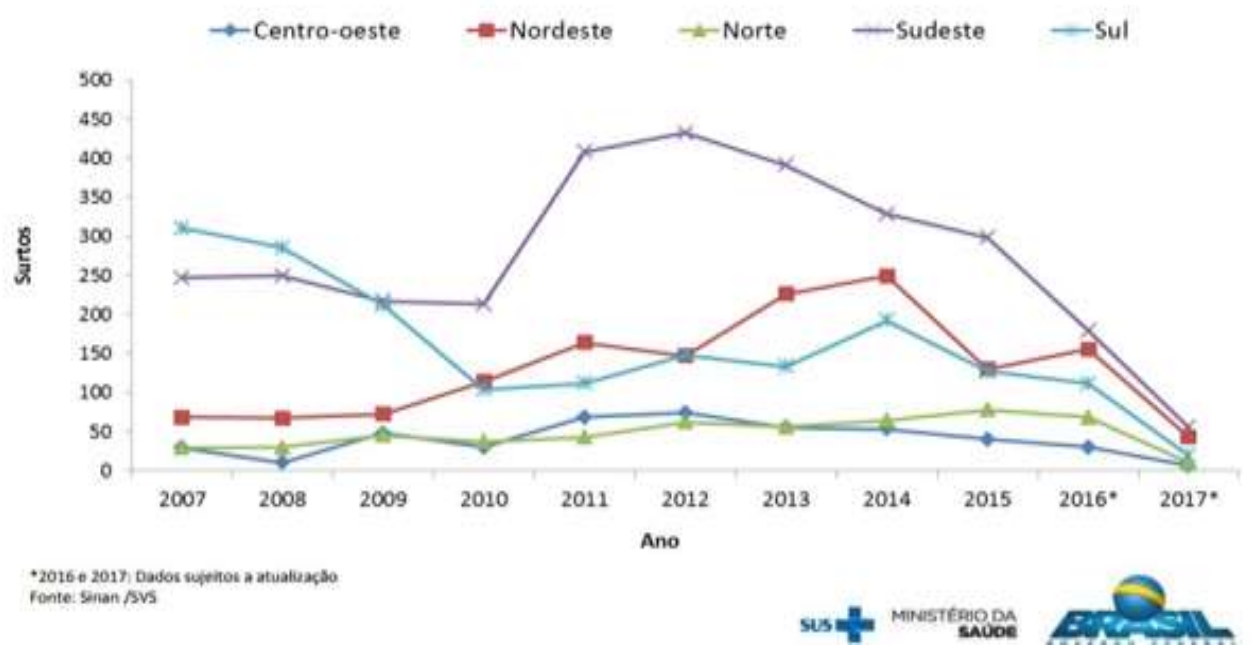

Fonte: Ministério da Saúde. Manual Integrado de Vigilância, Prevenção e Controle de Doenças Transmitidas por Alimentos, (2017).

O perfil epidemiológico das DVA ainda é pouco conhecido no Brasil porque somente alguns países e estados disponibiliza as estatísticas e casos com os dados referentes aos alimentos e agentes etiológicos envolvidos e os fatores contribuintes. De acordo com Carmo e colaboradores, os surtos de DVA podem ser investigados através da identificação etiológica laboratorial, exames clínicos, bromatológicos ou por critérios epidemiológicos. Por esses métodos é possível obter conclusões sobre seus agentes etiológicos, veículo, local de ocorrência e demais características pertinentes.

Entre alguns dos fatores que contribuem para o aumento do registro dessas doenças pode-se destacar: a) o aumento da população, b) o aumento de grupos populacionais vulneráveis ou mais expostos, c) o processo de urbanização, muitas vezes, desordenado, d) a produção e consumo de alimentos em condições inadequadas, e) o aumento da produção de alimentos e do comércio internacional, f) a melhoria dos sistemas de vigilância epidemiológica e, g) a melhoria dos métodos de diagnóstico e estrutura laboratorial para análises. Além desses fatores, podem ser incluídas outras causas que colaboram de forma menos expressiva para o aumento da ocorrência das DTA, como por exemplo, a utilização de novas modalidades de produção, o 
aumento no uso de aditivos, mudanças de hábitos alimentares, alterações climáticas e ambientais, a globalização e as facilidades atuais de deslocamento da população, em nível nacional e internacional (Ministério da saúde, 2010).

\subsection{Micro-organismos veiculados aos alimentos}

Gráfico 12: Principais micro-organismos em Surtos de DVA no Brasil, 2016.

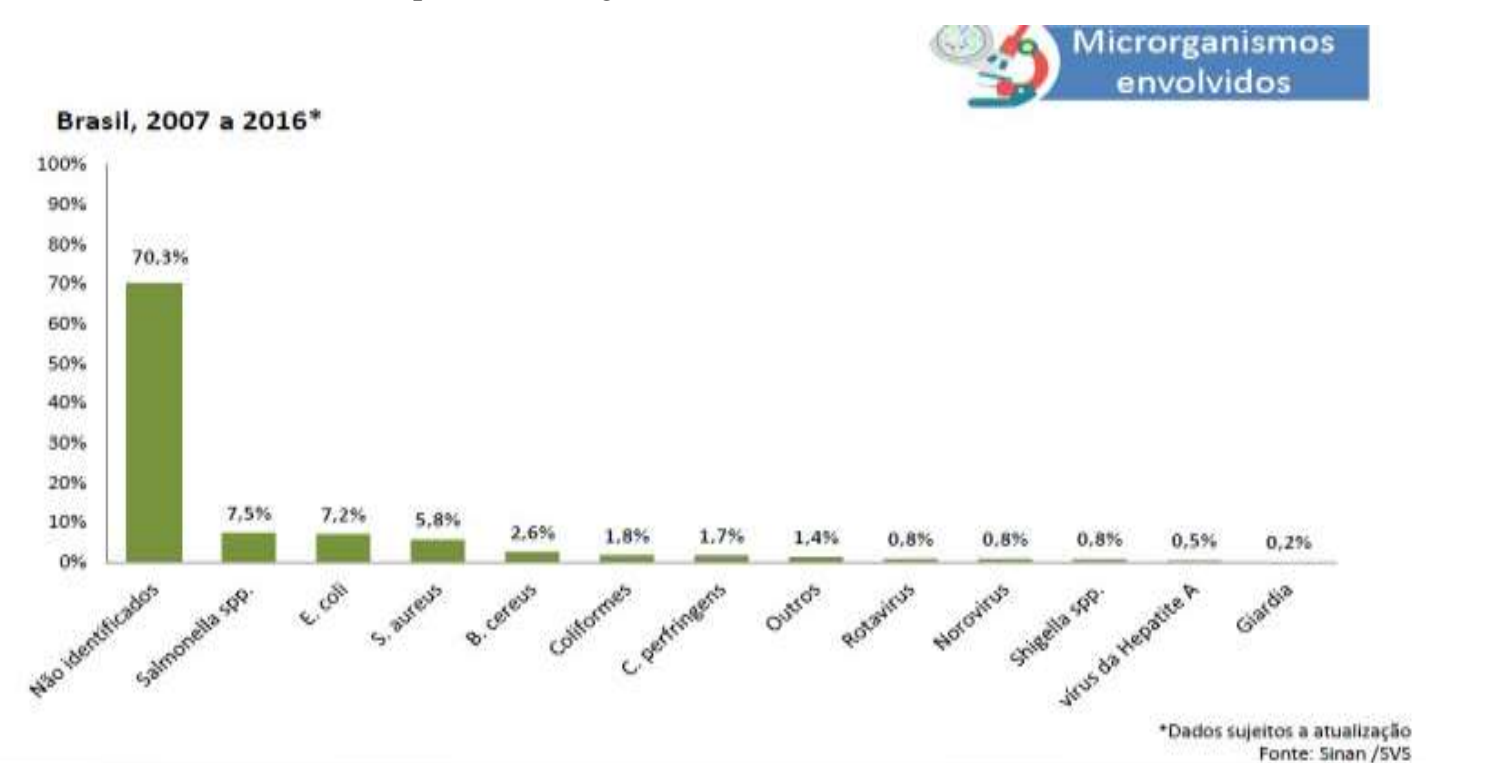

Fonte: Ministério da Saúde. Manual Integrado de Vigilância, Prevenção e Controle de Doenças Transmitidas por Alimentos, (2016).

Gráfico 13: Principais micro-organismos em Surtos de DVA no Brasil, 2017.

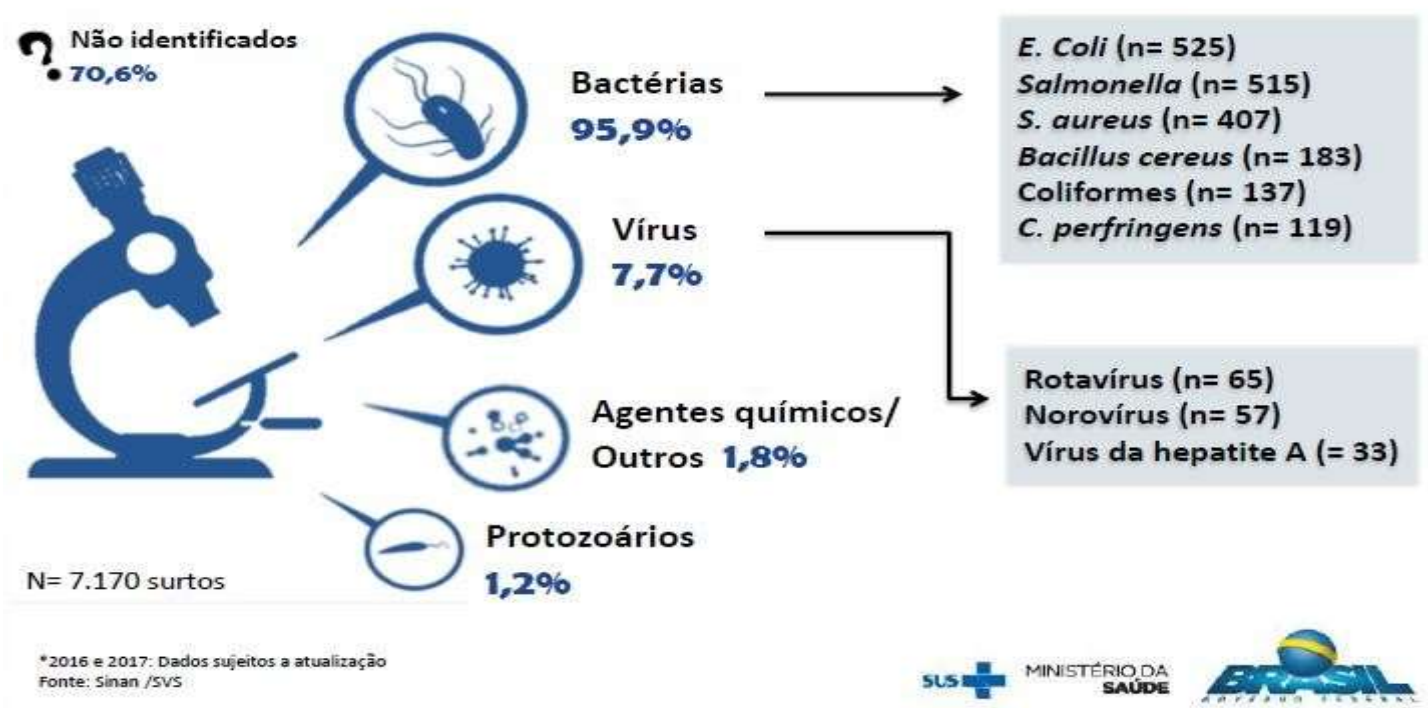

Fonte: Ministério da Saúde. Manual Integrado de Vigilância, Prevenção e Controle de Doenças Transmitidas por Alimentos, (2017).

A falta de higiene dos manipuladores durante o preparo e manipulação, bem como as condições de armazenamento do produto e os fatores intrínsecose extrínsecos podem ocasionar deteriorações no alimento ou DVA nos consumidores (Seixas, 2008). Portanto, podem contribuir para a maionese caseira e a industrializada estar frequentemente envolvida com surtos de origem alimentar em vários países (Di pinto, Novello, Montemurro, Bonerba, \& Tantillo, 2010; Norton et al., 2012). 
Os ovos e os produtos à base de ovos crus ou mal cozidos constituem- se nos principais alimentos veiculadores de Salmonella enteritidis e, consequentemente, causadores de infecções em humanos (Barancelli, Martin, \& Porto, 2012)

Associada aos surtos de DVA são os produtos cárneos e derivados. Segundo Welker et al., (2010) os produtos cárneos apresentam condições favoráveis à proliferação de micro-organismos devido à variedade de nutrientes, umidade e à baixa acidez. Além disso, as carnes podem ser facilmente contaminadas durante seu processo de produção como: o abate do animal, evisceração, manipulação no processamento e estocagem inapropriada.

\section{Conclusão}

Existe a dificuldade de registro das DVA, uma problemática mundial, com base nos dados analisados, apenas uma pequena parcela dos casos de DVA estão registrados nos bancos oficiais dos sistemas da Vigilância Sanitária, evidenciando o problema mundial de subnotificação.

Os surtos registrados geralmente envolvem um maior número de pessoas ou aqueles que apresentam sintomas mais prolongados ou severos. Apesar desses entraves, foi permitido identificar características importantes dessas síndromes como os principais agentes etiológicos, os alimentos mais comumente implicados, os fatores causais mais frequentes, as regiões e os sintomas mais evidentes.

Com base nesses dados, micro-organismos como Salmonella spp., S. aureus, B. cereus e E. coli foram agentes bacterianos importantes nas DVA ocorridas em diferentes países, enquanto L. monocytogenes parece ser a principal responsável pelos óbitos relacionados as DVA ocorridas no Brasil.

Os alimentos mais frequentemente envolvidos com as DVA foram os alimentos mistos, a água, leite e derivados, principalmente àqueles a base de ovos e produtos cárneos. Os principais fatores causais foram a manipulação inadequada de alimentos, a exposição prolongada dos alimentos à temperaturaambiente, a refrigeração e a cocção inadequadas dos alimentos, enquanto os restaurantes comerciais e as residências foram os locais de ocorrência de surtos mais frequentemente citados em diversos estudos.

Contudo, as unidades produtoras de alimentos e os moradores de residências deverão ser instruídos para ocorrer uma melhor ação e minimização desse problema mundial de saúde pública.

Nessa perspectiva, sugere-se a proposição de ações e estudos em termos de Políticas Públicas, que apontem para inclusão destes moradores de residências, em termos de manipulação e a inserção da Nutricionistas com vistas em todas as unidades comerciais, industriais produtoras e de manipulação a atribuir-lhes uma melhor qualidade e uma condição digna para o consumo.

\section{Referências}

Alves, E., Giarreta, A. G., \& Costa, F. (2012). Higiene pessoal dos manipuladores de alimentos dos Shoppings Centers da região da grande Florianópolis. Revista Técnico Científica do IFSC, 1, 604.

Barancelli, G. V., Martin, J. G. P., \& Porto, E. (2012). Salmonella in eggs: relation between production and safe consumption. Segurança Alimentar e Nutricional, 19, 73-82.

Buzby, J. C., \& Roberts, Tanya. (2009). The economics of enteric infections: human foodborne disease costs. Gastroenterology, 136, 1851-1862.

Câmara, F. M., Gomes, C. B., Matuk, T. T., \& Szarfarc, S. C. (2014). Caracterização dos resíduos gerados na Ceasa paulistana sob a ótica da saúde ambiental e segurança alimentar. Segurança Alimentar e Nutricional, 21(1): 395-403.

Capalonga, R., Ramos, R. C., Both, J. M. C., Soeiro, M. L. T., Longaray, S. M., Haas, S., \& Tondo, E. C. (2014). Salmonella serotypes, resistance patterns, and food vehicles of salmonellosis in southern Brazil between 2007 and 2012. The Journal of Infection in Developing Countries, 8, 811-817.

Guilherme, D. L. \& Esteves, D. C. (2017). Doenças transmitidas por alimentos e água. Conexão. Eletrônica, 14, 390-401. Gyles, C. L.

Di Pinto, A., Novello, L., Montemurro, F., Bonerba, E., \& Tantillo, G. (2010). Occurrence of Listeria monocytogenes in ready - to-eat foods from supermarkets in Southern Italy. New Microbiol., 33, 249 - 252, 
Fischer, M. M. (2013). Contaminação microbiológica de alimentos envolvidos em surtos de doenças transmitidos por alimentos ocorridas no estado do Rio Grande do Sul entre 2004 e 2012. 41 f. Monografia (Graduação) - Universidade Federal do Rio Grande do Sul.

Forsythe, S. J. (2010). Microbiology of Safe Food. (2a ed.), Oxford: BlackwellPublishing Ltda.

Gantois, I., Ducatelle, R., Pasmans, F., Haesebrouck, F., Gast, R., Humphrey, T. J., \&Van immerseel, F. (2009). Mechanisms of egg contamination bySalmonella Enteritidis. FEMS Microbiol., 33, 718 - 738.

Júnior, S. et al. (2008). Manual de controle higiênico-sanitário em serviços de alimentação. In: Manual de controle higiênico-sanitário em serviços de alimentação. (6a ed.), Ed Varela.

Klein, L. R., Bisognin, R. P., \& Figueiredo, D. M. S. (2017). Estudo do perfil epidemiológico dos surtos de doenças de transmissão hídrica e alimentar no rio grande do sul: uma revisão dos registros no Estado. Hygeia, 13, 48-64.

Marchi, D. M., Baggio, N., Teo, C. R. P. A., \& Busato, M. A. (2011). Ocorrência de surtos de doenças transmitidas por alimentos no Município de Chapecó, Estado de Santa Catarina, Brasil, no período de 1995 a 2007. Epidemiologia e serviços de saúde, 20(3), 401-407.

Mello, A. G., Gama, M. P., Marin, V. A., \& Colares, T. L. G. (2010). Conhecimento dos manipuladores de alimentos sobre boas práticas nos restaurantes públicos populares do Estado do Rio de Janeiro. Braz J Food Technol. 13(1): 60-8.

Melo, E. S., Amorim, W. R., Pinheiro, R. E. E., Corrêa, P. G. N., Carvalho, S. M. R., Santos, A. R. S. S., Barros, D. S., Alencar, E. T., Oliveira, C., Mendes, C. A., \& Sousa, F. V. (2018). Doenças transmitidas por alimentos e principais agentes bacterianos envolvidos em surtos no Brasil: revisão. PubVet, 12(10), 1-9

Ministério da Saúde. (2010). Portaria $n^{\circ}$ 2.472, de 31 de Agosto de 2010.Dispõe a relação de doenças, agravos e eventos em saúde pública denotificação compulsória em todo o território nacional e estabelecer fluxo,critérios, responsabilidades e atribuições aos profissionais e serviços de saúde.

Ministério da Saúde. (2016). Surtos de Doenças Transmitidas por Alimentos no Brasil.

Ministério da Saúde. (2008). Análise epidemiológica dos surtos de doenças transmitidas por alimentos no Brasil.

Ministério da Saúde. (2010). Secretaria de Vigilância em Saúde.Departamento de Vigilância Epidemiológica. Manual integrado de vigilância, prevenção e controle de doenças transmitidas por alimentos. Brasília:Editora do Ministério da Saúde.

Mürmann, L., Santos, M. C., Longaray, S. M., Both, J. M. C. \& Cardoso, M. (2008). Quantification and molecular characterization of Salmonella isolated from food samples involved in salmonellosis outbreaks in Rio Grande do Sul, Brazil. Brazilian Journal of Microbiology, 39: 529-534.

Nascimento, C. B. (2013). Surtos de Toxinfecção alimentar notificados e investigados no município de Porto Alegre no período de 2003 a 2011. 39f. Dissertação (Mestrado). Universidade Federal do Rio Grande do Sul.

Norton, S., Huhtinen, E., Conaty, S., Hope, K., Campbell, B., Tegel, M., Boyd, R., \& Cullen, B. (2012). A large point - sourceoutbreakof Salmonella Typhimurium linked to chicken, pork and salad rolls from aVietnamese bakery in Sydney. Western Pac urveill Response J., 21, 16-23.

Rouquayrol, M. Z. \& Silva, M. G. C. (2018). Epidemiologia \& saúde. Medbook.

Pereira, A. S., Shitsuka, D. M., Parreira, F. J. \& Shitsuka, R. (2018). Metodologia Da Pesquisa Científica. UFSM

Santos, E. A. (2014). Implantação de Ferramentas de Gestão da Qualidade dos Alimentos em uma Unidade de Alimentação e Nutrição Institucional: um estudo de caso. 161 f. Dissertação (Mestrado). Instituto Federal do Triângulo Mineiro, UBERABA - MG.

Seixas, F. R. F. (2008). Verificação das Boas Práticas de Fabricação (BPF) e Análise da Qualidade Microbiológica de Saladas Adicionadas de Maionese Comercializadas na Cidade de São José do Rio Preto - SP. (Dissertação de Mestrado) UNESP-Campus de São José do Rio Preto.

Sirtoli, D. B., \& Comarella, L. (2018). O papel da vigilância sanitária na prevenção das doenças transmitidas por alimentos (DTA). Revista Saúde e Desenvolvimento, 12(10), 197-209.

Soares, L. S., Almeida, R. C. C., Cerqueira, E. S., Carvalho, J. S., \& Nunes, I. L. (2012). Knowledge attitudes and practices in food safety and the presence of coagulase-positive staphylococci on hands of food handlers in the schools of Camaçari, Brazil. Food Control; 27(1): 206-13.

Terres, A., Pereira, \& Bissigo. F. (2015). Perfil epidemiológico de surtos veiculados por alimentos na Serra Gaúcha/RS. In: Congresso de Pesquisa e Extensão da Faculdade da Serra Gaúcha. 2015. 852-854.

Tokuç, B., Ekuklu, G., Berberogu, U., Bilge, E., \& Dedeler, H. (2009). Knowledge, attitudes and self-reported practices of food service staff regarding food in Edirne,Turkey. Food Control. 20(6): 565-8.

Wagner, V. R., Silveira, J. B., \& Tondo, E. C. (2014). Salmonellosis in Rio Grande do SulState, Southern Brazil, 2002 to 2004. BrazilianJournal of Microbiology. 44, 723-729.

Welker, C. A. D., Both, J. M. C., Longaray, S. M., Haas, S., Soeiro, M. L. T., \& Ramos, R. C.(2010). Análise microbiológica dos alimentos envolvidos em surtos de doenças transmitidas por alimento (DTA) ocorridos no estado do Rio Grande do Sul, Brasil. Revista Brasileira de Biociências, 8, $44-48$.

Who - World Health Organization. (2019). World health statistics 2019: monitoring health for the SDGs, sustainable development goals. Geneva: World Health Organization.

Zhang, G., Brown, E. W., \& Hammack, T. S. (2013). Comparison of different preenrichment broths, egg: preenrichment broth ratios, and surface disinfection for the detection of Salmonella enterica ssp. enterica serovar Enteritidis in shell eggs. Poult Sci., 92, 3010 - 3016. 